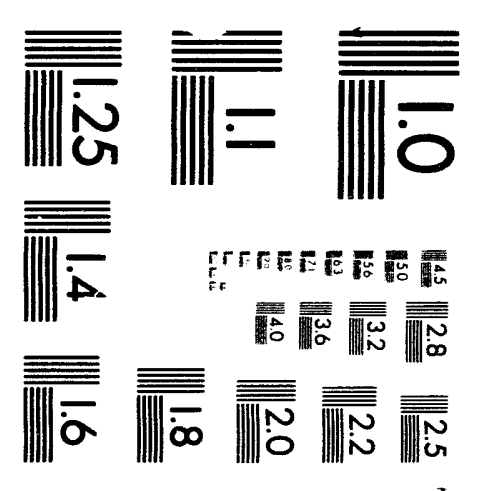



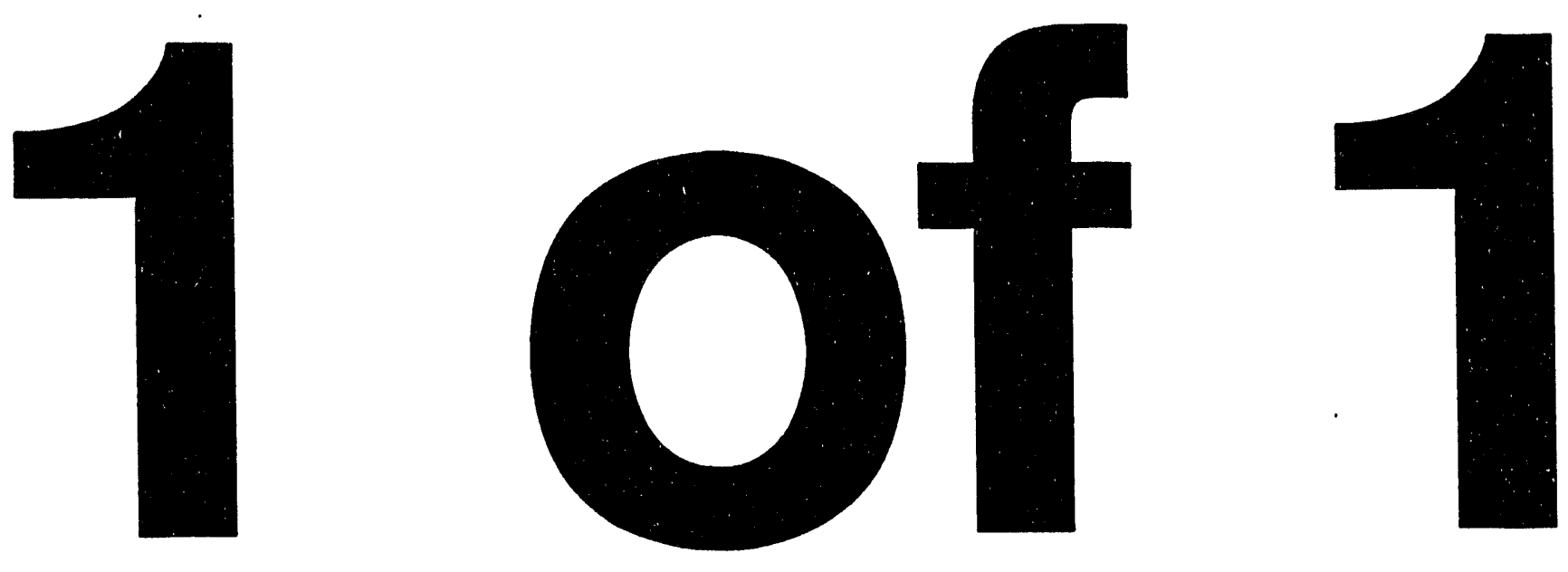


\section{Conf-930511--502}
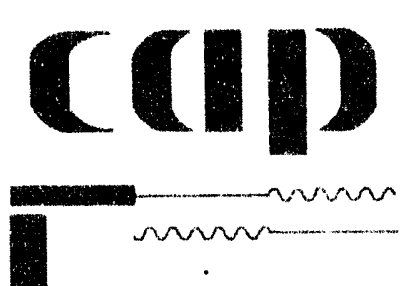

Effects of the Third Order

BNL-49312

CAP 102-93C

\section{Transfer Maps and Solenoid on \\ a High Brightness Beam}

Zohreh Parsa

Physics Department, 901A

May 1993

\section{CENTER FOR ACCELERATOR PHYSICS}

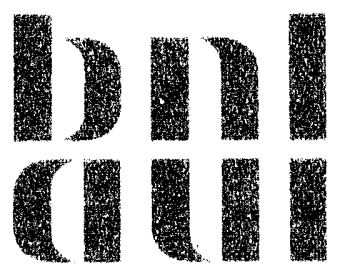

BROOKHAVEN NATIONAL LABORATORY ASSOCIATED UNIVERSITIES, INC. 


\section{DISCLAIMFR}

This report was prepared as an account of work sponsored by an agency of the United States Government. Neither the United States Government nor any agency thereof, nor any of their employees, nor any of their contractors, subcontractors, or their employees, makes any warranty, express or implied, or assumes any legal liability or responsibility for the accuracy, completeness, or usefulness of any information, apparatus, product, or process disclosed. or represents that its use would not infringe privately owned rights. Reference herein to any specific commercial product, process, or service by trade name, trademark, manufacturer, or otherwise, does not necessarily constitute or imply its endorsement, recommendation, or favoring by the United States Government or any agency, contractor or subcontractor thereof. The views and opinions of authors expressed herein do not necessarily state or reflect those of the United States Government or any agency, contractor or subcontractor thereof. 


\title{
Effects of the Third Order Transfer Maps and Solenoid on a High Brightness Beam
}

\author{
Zohreh Parsa \\ Physics Department \\ Brookhaven National Laboratory, \\ Upton, NY 11973
}

\section{Abstract}

We present a sketch of the formulation for obtaining Lie algebraic transfer maps for the solenoid through third order and its effect on the beam of charged particles. We discuss simulation results showing effects of solenoids on the laser driven high brightness photoelectrons for the proposed alternate injection system for Brookhaven Accelerator Test Facility.

\section{INTRODUCTION}

A brief overview of a Lie algebraic formulation is given in section II. Using Hamiltonian dynamics we describe the motion of a charged particle through electromagnetic fields. With Lie transformations we obtain the maps and trajectories for a particle along the beamline in a magnetic field (e.g. of solenoid). We discuss the transfer maps for magnetic elements and solenoid through third order and their effects on the beam of charged particles. In section III we discuss the effects of the solenoids used in the design of the proposed alternate injection system for the Brookhaven Accelerator Test Facility (ATF) [1].

\section{FORMALISM}

In this section we present an overview of the formalism used to obtain the trajectories of a particle along the beamline via Lie transformation. Using Maxwell's equations, axisymmetrical fields, and the relativistic equations for the charged particles motion along the beamline we can obtain the magnetic field components everywhere (e.g. of a solenoid given the on axis longitudinal component of the field $\left.B_{z}=(B, 0,0)\right)$ and its effect on the particles motion.

We express the canonical equations in $2 \mathrm{n}$-Dimensional phase space (e.g 6 Dim., in our calculation), as

$$
\frac{d \psi_{i}}{d t}=\left[\psi_{i}, H\right], \quad i=1,2, \ldots 2 n
$$

and in terms of the Lie transformations as

$$
\frac{d \psi_{i}}{d t}=-: H: \psi_{i}, \quad i=1,2, \ldots 2 n
$$

Where the Lie operator $(: H:)$ is generated by the Hamiltonian, (H), and Lie transformation,

$$
M=e^{-\ell: H},
$$

could generate the solution to Eq. (2) as

$$
\psi_{i}=M \psi_{i}(0)
$$

where $\psi_{i}$ is the value of $\psi_{i}(t)$ at $t>0$ and $\psi_{i}(0)$ is the initial trajectory. The interest is to find solutions to equations of motion which differ slightly from the reference orbit (e.g. the design orbit of an accelerator beamline. Design orbit for solenoid is along z-axis). Thus, we choose the canonical variables, from the values for the reference trajectory (for small deviations) and Taylor expand the Hamiltonian ( $\mathrm{H})$ about tine design trajectory:

$$
H=H_{2}+H_{3}+\ldots
$$

Where $H_{n}$ is a homogeneous polynomial of degree $\mathrm{n}$ in the canonical variables. After transformations to the normalized dimensionless variables, we obtain the effective Hamiltonian $H^{\mathrm{New}}$, expressed as

$$
H^{\mathrm{New}}=F_{2}+F_{3}+F_{4} \ldots
$$

Thus the particle trajectory $\vec{\psi}=\left(X, P_{X}, Y, P_{Y}, \tau, P_{\tau}\right)$ through a beamline element of length $L$ can be described by

$$
\psi_{i}^{f}=-: H^{\text {New }}: \psi_{i}, \quad i=1,2, \ldots 2 n
$$

The exact symplectic map that generates the particle trajectory through that element is,

$$
M=e^{-L: H^{\text {New }}},
$$

where, $M$ describes the particle behavior through the element of length $\mathbf{L}$. Using the factorization and expanding $H^{\mathrm{New}}$ as in Eq. (6), we obtain

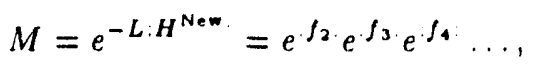

(for a map through 3rd order we need to include terms of $\mathfrak{F}, \mathfrak{B}$, and $\left.f_{4}\right)$. Where $f_{2}=L F_{2}, f_{3}=L F_{3}, f_{4}=L F_{4}$, etc.

To illustrate the above formalism, consider the evolution of the motion of particles in an external electromagnetic field described by the II amiltonian

$$
\begin{aligned}
H & =\sqrt{m^{2} c^{4}+c^{2}\left[\left(p_{x}-q A x\right)^{2}+\left(p_{y}-q A_{y}\right)^{2}+\left(p_{z}-q A_{z}\right)^{2}\right]} \\
& +e \phi(x, y, z ; t)
\end{aligned}
$$

where $m$ and $q$ are the rest mass and charge of the particle, $A$ and $\phi$ are the vector and scalar potentials such that $\vec{B}=\nabla \times \vec{A}, \vec{E}=-\nabla \phi-\nabla \vec{A} / \partial t$. 
Making a canonical transformation from $H$ to $H_{1}$ and changing the independent variable from time $t$ to $z$ (for convenience) for a particle in magnetic field (e.g. of solenoid) results in:

$$
p_{z}=\left[\left(p_{x}-q A x\right)^{2}+\left(p_{y}-q A_{y}\right)^{2}+p_{t}^{2} / c^{2}-m^{2} c^{2}\right]^{1 / 2} .
$$

Where $H=-p_{t}, H_{1}=-p_{z}$ and $t=\left(z / v_{0 z}\right)$ the time as a function of $z$. We next make a canonical transformation from $H_{1}$ to $H^{\mathrm{New}}$, with a dimensionless deviation variables (for convenience), $X=x / l, Y=y / l, \tau=c / l\left(t-z / v_{0 z}\right)$, $P_{x}=p_{x} / p_{0}, P_{y}=p_{y} / p_{0}, P_{T}=\left(p_{t}-p_{0} t\right) / p_{0} c$., where $l$ is a length scale (taken as $1 \mathrm{~m}$ in our analysis), with $\mathbf{P}=\vec{P} x+\vec{P} y$ and $\mathbf{Q}=\vec{X}+\vec{Y}$ defined as two dimensional vectors [3]. $p_{0}$ and $p_{0} c$ are momentum and energy scales. Where $p_{0}$ is the design momentum, $v_{0 z}$ is the velocity on the design orbit and $p_{0 t}$ is value of $p_{t}$ on the design orbit $\left(p_{0 t}=\sqrt{m^{2} c^{4}+p_{0}^{2} c^{2}}\right)$ (reminding that design orbit for the solenoid is along the $z$-axis).

Thus, expanding the new Hamiltonian (eq.(6)) leads to:

$$
\begin{gathered}
F_{2}=\frac{P_{\tau}^{2}}{\left(2 \beta^{2} \gamma^{2}\right)}-\frac{1}{2} B_{0}(\vec{Q} \times \vec{P}) \cdot \hat{z} \\
+\frac{1}{8} B_{0}^{2} Q^{2}+\frac{P^{2}}{2} \\
F_{3}=\frac{P_{\tau}^{3}}{\left(2 \beta^{3} \gamma^{2}\right)}-\frac{P_{\tau}}{2 \beta} B_{0}(\vec{Q} \times \vec{P}) \cdot \hat{z} \\
+\frac{P_{\tau}}{8 \beta}\left(B_{0}^{2} Q^{2}+4 P^{2}\right) \\
F_{4}=\frac{P_{\tau}^{4}\left(5-\beta^{2}\right)}{8 \beta^{4} \gamma^{2}}+\frac{P_{\tau}^{2} Q^{2} B_{0}^{2}\left(3-\beta^{2}\right)}{16 \beta^{2}} \\
-\frac{P_{\tau}^{2}}{2}(\vec{Q} \times \vec{P}) \cdot \hat{z} \frac{B_{0}\left(3-\beta^{2}\right)}{2 \beta^{2}} \\
+\frac{P_{\tau}^{2}}{2} \frac{P^{2}\left(3-\beta^{2}\right)}{2 \beta^{2}}+\frac{Q^{4}}{16}\left(B_{0}^{4}-4 B_{0} B_{2}\right) / 8 \\
+\frac{Q^{2}}{4} \frac{P^{2} 3 B_{0}^{2}}{4}+\frac{Q^{2}}{4}(\vec{Q} \times \vec{P}) \cdot \hat{z}\left(B_{2}-B_{0}^{3}\right) / 4 \\
-\frac{1}{8}(\vec{P} \cdot \vec{Q})^{2} B_{0}-\frac{P^{2}}{4}(\vec{Q} \times \vec{P}) \cdot \hat{z} B_{0}+\frac{P^{4}}{8}
\end{gathered}
$$

Following the hamiltonian flow generated by $H^{\text {New }}=$ $F_{2}+F_{3} \ldots$ from some initial $\psi_{0}$ to a final $\psi_{f}$ coordinates we can calculate the transfer map $M$ (eq. 9) for the solenoid. Where $F_{2}, F_{3}$, and $F_{4}$ would lead to the 1st, 2nd, and 3rd order maps. The effects of which can be seen from eqs. (12-14). For example, the 2nd order effects due to solenoid transfer maps are purely chromatic aberrations (eq. 13). In addition to chromatic effects, we note the third order geometric aberrations (eq. 14). The coupling between $X, Y$ planes produced by a solenoid is rotation about the $z$-axis which is a consequence of rotational invariance of the Hamiltonian $H^{\mathrm{New}}$ shown by eqs.(12-14), due to axial symmetry of the solenoid field.

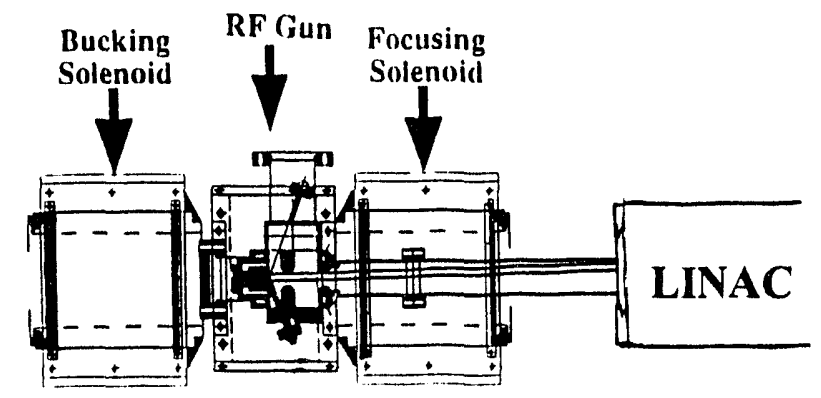

Figure 1: Sketch of the alternate injection system for ATF. A solenoid + gun + solenoid combination is placed straight ahead into the linac. (Not scaled).

For beam simulations, $M$ can be calculated to any order using numerical integration techniques such as RungeKutta method depending on the computer memory and space available [3].

\section{BNL ATF INJECTION SYSTEM}

In this section we present some of our calculations and simulation results obtained for the proposed alternate (straight-ahead) injection system which consists of a pair of solenoids and an if gun placed directly into the linac $[1,7]$.

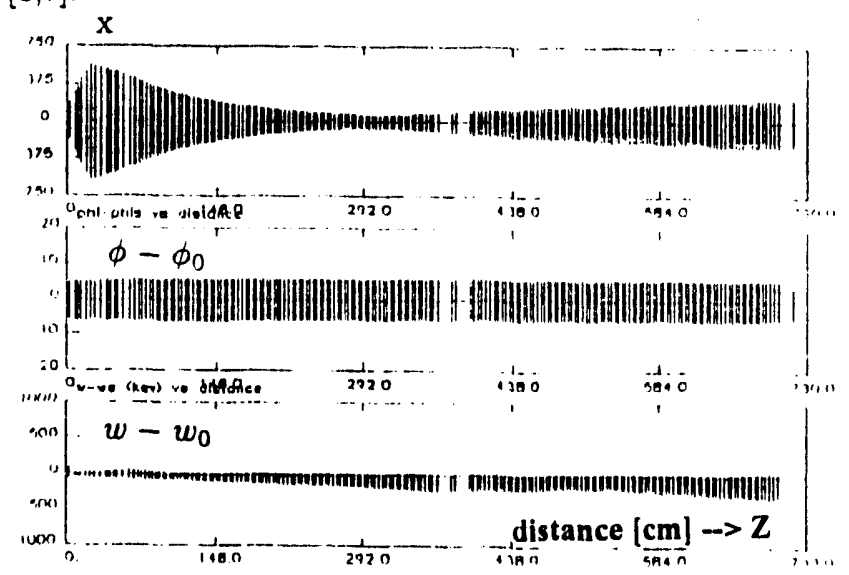

Figure 2: Shows the change in position $x[\mathrm{~cm}]$, phase $\phi-\phi_{0}$ [degree] and energy $w-w_{0}[\mathrm{KeV}]$ of particles at each element location, from the cathode through the linac exit. With solenoid current of $2140 \mathrm{~A}$ and $d=62 \mathrm{~cm}$ and $\sigma_{r}=.9 m m, \sigma_{z}=5 p s$.

Present injection system consists of 2 sets of quadrupole triplets and a $180^{\circ}$ achromatic double bend [1], where beam diverges quickly as it exits the gun and gets large as it traverses through the dipoles and the linac. We have used a pair of solenoids (placed before and after the gun such that $B=0$ on the cathode) shown in Fig. 1, which controls the beam divergence at the gun exit, reduces the emittance dilution due to space charge forces, and improves the conditions for production of high brightness low emittance beam needed e.g., for Free Electron Laser. and Inverse Free Electron Laser experiments. Figure 2 shows how the beam size increases as it drifts from the 


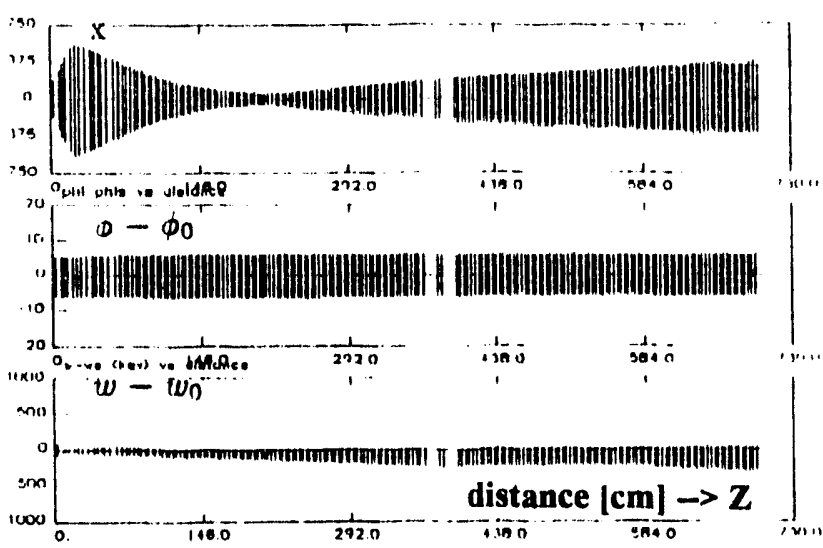

Figure 3: Shows the change in position $x[\mathrm{~cm}]$, phase $\Phi-\dot{\phi}_{0}$ [degree] and energy $w-w_{0}[\mathrm{KeV}]$ of particles at each element location, from the cathode through the linac exat. With solenoid current of $2180 \mathrm{~A}$ and $d=62 \mathrm{~cm}$ and $\sigma_{r}=.9 \mathrm{~mm}, \sigma_{z}=5 p s$.

gun into the linac. The Beam converges to a waist in the linac resulting in a beam of smaller emittance at the linac exit which is of interest at ATF.

Comparison of Figs. 2 and 3 show locations of the beam waist and beam envelopes along the beamline from cathode through the linac. In Fig. 3, a $2 \%$ increase in the solenoid current resulted in the shift in the position of the beam waist in the linac and an emittance increase from $\left(\epsilon_{x}^{\mathrm{V}, r m s}=.278, \epsilon_{y}^{\mathrm{V}, r m s}=.243\right)$ to $\left(\epsilon_{x}^{\mathrm{V}, r m s}=.390\right.$, $\epsilon_{y}^{\mathrm{V} . r m s}=.333$ ) at the linac exit (as compared to Fig. 2). This illustrates the effects due to variation of the solenoid strength on the beam dynamics. In this analysis we used an initial $E=100 \mathrm{MV} / \mathrm{m}$ on the cathode, laser pulse length $\left(2 \sigma_{z}\right)$ of $10 \mathrm{ps}$, spot size $\sigma_{r}=0.9 \mathrm{~mm}$. initial phase of $43^{\circ}$. and $d=\delta 2 \mathrm{~cm}$, (the distance from :athode to linac entrance). The solenoid for this design can vary up to $4.0 \mathrm{KG}$ in strength. For example with a $2.2 \mathrm{KG}$ solenoid strength we can preserve the beam quality and achieve high brightness, low emittance beam at the linac exit, which is needed for the FEL and laser acceleration experiments at ATF. With program PARMELA [6], with $\sigma_{r}=1 \mathrm{~mm}$ and $0.9 \mathrm{~mm}$ (uniform beam distribution) we sbtained beam emittance of lew ienths of $\mathrm{cm}$-mrad with energies of about $46 \mathrm{MeV}$ and brightness $\left(B=\left[^{\text {peak }} / \pi \epsilon_{x}^{V} \epsilon_{y}^{i V}\right)\right.$ of orders of $10^{13}$ for the beam emerging from the exit of the linac.

\section{SUMMARY}

We presented a sketch of the formalism used to obain Lie algebraic maps through third order tor magnetic rlements e.t. a solenold. Vote that, the 2nd order aberration due to solenold is purely chromatic. We discussed rfects sf solenoids used in the design of the alternate injection system for the ATF ac Brookhaven Vational Lab. Where a pair of solenoids and an of gun is placed directly ato the linac, to improve the beam loss and the emittance. growth at the linac exut (as may be with the present louve bend iystem/. With iolenold+yun+solenold itraisht injection into the linac scheme we reduce the emittance dilution due to space charge forces, and produce the beam needed for FEL, IFEL and other laser acceleration experiments. We obtained small emittance (few tenths of $\mathrm{cm}-\mathrm{mrad})$ and high brightness of orders oi $10^{13}$. The solenoids used in the alternate injection system. controls the beam divergence at the gun exit, reduces the emittance dilution due to the space charge forces on the beam and produces a smaller beam emittance needed for the experiments at $\mathrm{ATF}$.

\section{REFERENCES}

*Work performed under the auspices of the U.S. Department of Energy, Contract Number DE-ACO2-i6-CH000l6.

1. Z. Parsa, "Development of the Alternate Entry Port for the $A T F^{n}$, Proc. [EEE May 17-20 PAC, Washington D.C. (1993).

2. Z. Parsa, 'Lie Algebraic Transfer . Maps of . Magnet System and Vonlinear Aberrations in the Beam". BNL Tech. Note ADD/CAP/TN j (1/21/89) and Reierences therein. Additional Lie transfers maps for drifts. quadrupole. dipole etc. were included in this reference.

3. A. Dragt, Program MARYLIE, Private comm.; ibid *Vumerical third-order transfer map for solenoud " VIM, A298 (1990), and References therein. MARYLIE includes 3rd order maps for solenoid with soit-edge "bump" function model for the field. We thank A.Dragt for discussions and providing his code. We did not include any results with MARYLIE for our analysis of the ATF alternate injection system with solenoids, since this code does not provide acceleration through the if gun and the linac. Results with MARYLIE for the beamline without if and linac accelerations will be given in a separate note.

t. Z. Parsa, "Prelimanary Emattance Measurements and Calculations of the Laser Excrted Beam from the BNL Accelerator Test Facrlity Radio Frequency ElecIron Gun". Vol.II, Sec.II. pl-j4, BNL-.j2292 (1991) and References therein: K. VicDonald st al. Proc.. EPAC88, June (1988); K. Batchelor th al. Operatzonal Status of Brookhaven tccelerator Test Faczlity" Proc. 1989 IEEE Particle Accelerator Conf. Chicago. III, March 20-23. 1989. \#89(2H2669-1) (1990).

j. Z. Parsa. "On Beam Emittance ind Invariants - Applications to tTF Beamline". Proc. 'nd EPAC. Vice France. Vile Ed.. Frontiers. (1990); BNL Report BNL-44364 (1990) and Ref, there!n.

i. Z. Parsa. and L. Young, -The BNL Photocathode. Gun with the Code P.AR.MELA". Vuct. Inst. : Meths. A318; BNL_16804 (1991): L. Young, (Privace omms. , P.1RMELA , 199:3 version.

$i$ 7. Parsa. In tlternate Injectzon siystem for the $3 \mathrm{VL}$

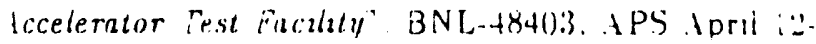
15. Wasnington D ( $\therefore$ : 1993:3). 

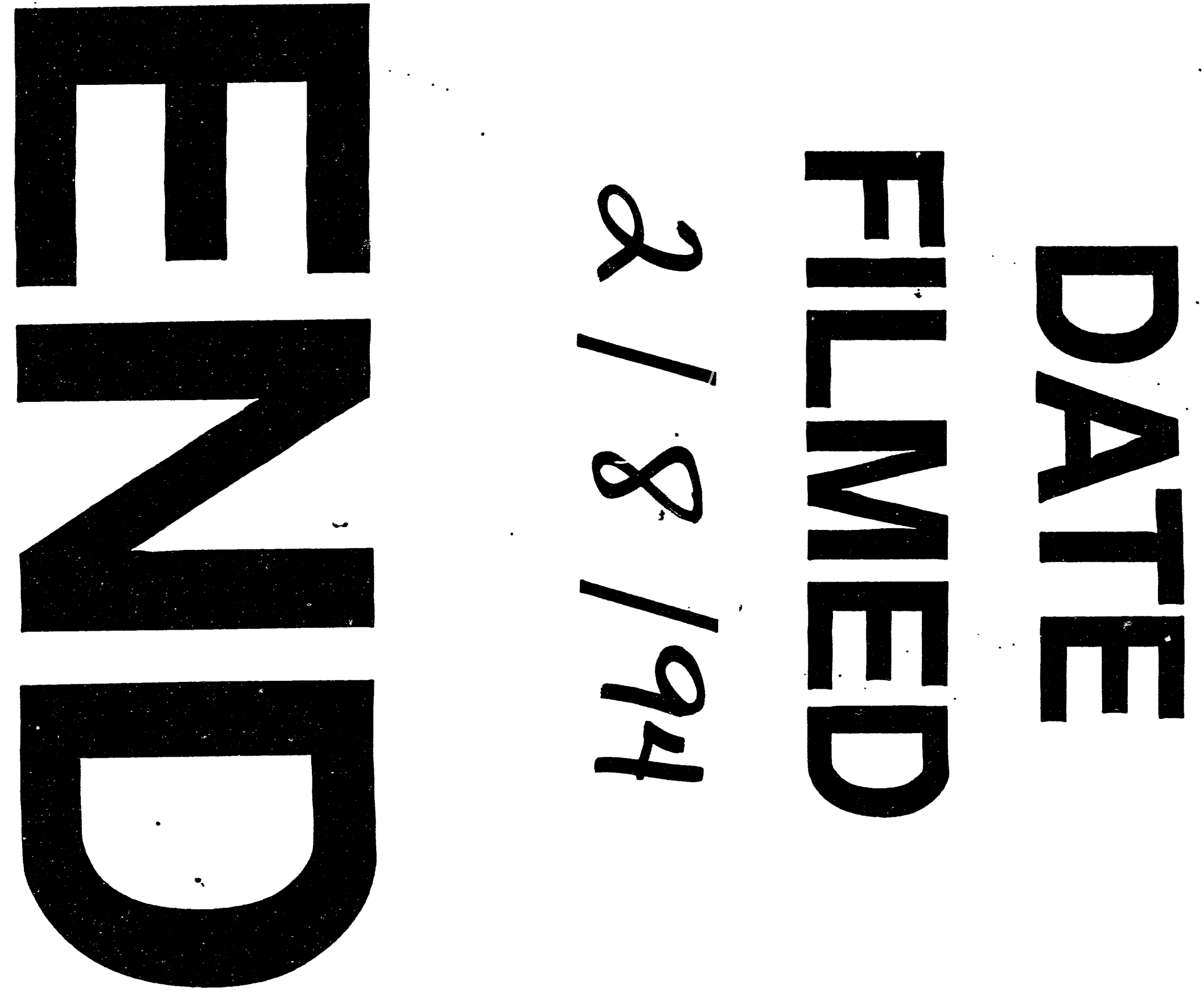
